\title{
Norois
}

Environnement, aménagement, société

\section{Diversités démographiques et migrations résidentielles dans les espaces ruraux en Wallonie (Belgique)}

Demographic diversities and residential migration in rural spaces in Wallonia (Belgium)

Thierry Eggerickx, Jean-Paul Sanderson, Amel Bahri et Jean-Pierre Hermia

\section{OpenEdition}

\section{Journals}

Édition électronique

URL : http://journals.openedition.org/norois/1294

DOI : $10.4000 /$ norois. 1294

ISBN : 978-2-7535-1553-6

ISSN : $1760-8546$

Éditeur

Presses universitaires de Rennes

\section{Édition imprimée}

Date de publication : 1 décembre 2007

Pagination : 117-136

ISBN : 978-2-7535-0616-9

ISSN : 0029-182X

Référence électronique

Thierry Eggerickx, Jean-Paul Sanderson, Amel Bahri et Jean-Pierre Hermia, « Diversités démographiques et migrations résidentielles dans les espaces ruraux en Wallonie (Belgique) », Norois [En ligne], 205 | 2007/4, mis en ligne le 01 décembre 2009, consulté le 30 avril 2019. URL : http:// journals.openedition.org/norois/1294; DOI : 10.4000/norois.1294 


\title{
DIVERSITÉS DÉMOGRAPHIQUES ET MIGRATIONS RÉSIDENTIELLES DANS LES ESPACES RURAUX EN WALLONIE (BELGIQUE)
}

\author{
Thierry Eggerickx, Jean-Paul Sanderson, Amel Bahri, Jean-Pierre Hermia \\ Gédap (Groupe d’Étude de Démographie Appliquée) \\ (Université catholique de Louvain), \\ Département des Sciences de la Population et du Développement (SPED) \\ Place Montesquieu 1 - 1348 LouvaIN-LA-Neuve (Belgique) \\ eggerickx@sped.ucl.ac.be, sanderson@sped.ucl.ac.be,bahri@sped.ucl.ac.be,hermia@sped.ucl.ac.be
}

\section{RÉSUMÉ}

Sur base d'un ensemble d'indicateurs sociaux, économiques, environnementaux. . et en utilisant des méthodes statistiques d'analyse en composantes principales et de classification, six types d'espaces ruraux ont pu être distingués en Wallonie. L'objectif était de vérifier si ces types d'espaces ruraux présentent des paramètres et des dynamiques démographiques particuliers. Les résultats ont démontré que l'univers rural wallon est marqué par la diversité des caractéristiques (répartition par âge, structure des ménages) et des comportements (mortalité, fécondité, migrations) démographiques. Certaines différences significatives, en termes de longévité ou encore d'importance relative de ménages monoparentaux, reflètent des inégalités sociales importantes et souvent insoupçonnées. Les migrations constituent le moteur de la démographie locale. Elles contribuent très largement à la croissance de la population des espaces ruraux wallons et, par leur sélectivité selon l'âge et le type de ménage, elles renforcent les contrastes sociodémographiques entre ceux-ci.

MotS CLÉ : Communes rurales - Démographie - Migration - Périurbanisation - Wallonie.

\section{ABSTRACT \\ Demographic diversities and residential migration in rural spaces in Wal- lonia (Belgium)}

On a basis of a set of social, economic and environmental indicators, and by using multivariate analysis, six types of rural spaces have been distinguished in Wallonia. The objective was to check if these types of rural spaces convey particular demographic profiles and trends. The results show indeed that the Walloon rural area is rather heterogeneous as regards characteristics such as age and household distributions; and demographic behaviours (mortality, fertility, migrations). Some significant differences, in terms of longevity or from the relatively high proportion of single-parent households, reflect important and often unsuspected social disparities. This paper also demonstrates that on local scale, and in a context of land pressure with saturated real estate markets, migrations contribute to increase stronger social and demographic disparities within rural areas in Wallonia.

KEY WORDS : Demography - Migrations - Periurbanisation - Rural Communes - Wallonia. 
Depuis le milieu des années 1950, l'exode urbain - au départ de l'agglomération de Bruxelles - s'est assorti d'une croissance démographique importante des communes rurales environnantes de la province du Brabant Wallon. Ce phénomène de périurbanisation, qui concerne surtout des jeunes ménages aux revenus moyens et élevés, participe à la redynamisation démographique de ces espaces. A côté de ces communes périurbaines, d'autres communes rurales connaissent des dynamiques différentes (Oris, 2000; Eggerickx et Capron, 2001) dans un contexte global de reprise démographique commun à de nombreux territoires ruraux d'Europe occidentale (Champion, 2000; Datar, 2003; Luginbühl, 2007; Perrier-Cornet, 2003). Les unes accueillent ceux qui ne peuvent rester ou s'établir en zone périurbaine et d'autres, des personnes plus âgées, ayant atteint l'âge de la retraite et cherchant un cadre de vie plus agréable et plus conforme à leurs aspirations (Eggerickx et al., 2002 b).

La plupart des études démographiques récentes se focalise sur les caractéristiques et les comportements de la population des agglomérations urbaines et de leur proche périphérie. Les espaces ruraux sont généralement négligés ou considérés comme un ensemble homogène (Houillon et Thomsin, 2000). En 1996, des géographes travaillant pour l'Institut National de Statistique (INS) ont publié une étude délimitant les régions urbaines de Belgique (Van der Haegen et al., 1996). Ces régions, au nombre de 17, représentent les principales zones de concentration de la population, des activités économiques et de la vie socioculturelle. Cette typologie des régions urbaines constitue une clé de lecture intéressante et pratique de l'univers communal belge, mais elle enferme le « reste des communes »- celles qui ne font pas partie de ces 17 régions urbaines - dans une catégorie résiduelle, a priori sans particularité.

Cette étude poursuit un double objectif. Il s'agira tout d'abord de caractériser sur la base d'indicateurs sociaux, économiques, environnementaux et de qualité de vie les communes de la Wallonie $^{1}$ (fig. 1) n'appartenant pas aux agglomérations urbaines. En utilisant une analyse en composantes principales et une analyse de classification, nous dégagerons des types de communes rurales. Ensuite, nous vérifierons si ces divers groupes se caractérisent par la convergence ou la divergence de leurs caractéristiques et comportements démographiques. Une importance particulière sera accordée à l'étude des migrations, lesquelles constituent le phénomène démographique de loin le plus influent à l'échelle locale.

\section{Diversité de l'espace rural wallon : élaboration d'une typologie des entités locales}

Pour définir le champ d'observation et identifier les communes composant l'espace rural wallon, nous avons utilisé la typologie des régions urbaines proposée par l'Institut National de Statistique (Van der Haegen et al., 1996). Celle-ci identifie 7 grandes régions urbaines en Wallonie (Charleroi, La Louvière, Liège, Mons, Namur, Tournai et Verviers), en fonction de critères tels que la concentration de la population, les activités économiques, les migrations pendulaires, le logement, etc. Au sein de ces régions urbaines, les agglomérations - soit 34 communes sur les 262 que compte la Wallonie - n'ont pas été prises en compte dans cette typologie des espaces ruraux ${ }^{2}$ (fig. 2).

Ayant ainsi délimité un cadre spatial, on peut à présent tenter d'en élaborer une typologie à partir d'une série d'indicateurs classés selon trois dimensions ${ }^{3}$ (tableau 1).

La dimension socio-économique : si les espaces périurbains se caractérisent par un niveau de vie assez élevé, il n'en va pas de même pour tout le milieu rural dont certaines entités peuvent connaître des situations de précarité. Nous avons sélectionné des variables telles que le revenu

1. La Belgique est scindée en trois grandes régions : Bruxelles-Capitale, la Flandre et la Wallonie.

2. Ces agglomérations urbaines figurent en blanc sur la carte de localisation (figure 2).

3. Il s'agit d'indicateurs statiques relatifs à une année donnée. Ce choix est motivé par deux raisons. D'une part, les dimensions environnement et isolement se basent sur des indices de satisfaction extraits du recensement de 2001. Il s'agit d'informations inédites qui ne sont donc pas disponibles au recensement de 1991. D’autre part, notre objectif est de présenter une photo à un moment donné de la diversité des espaces ruraux en Wallonie. 


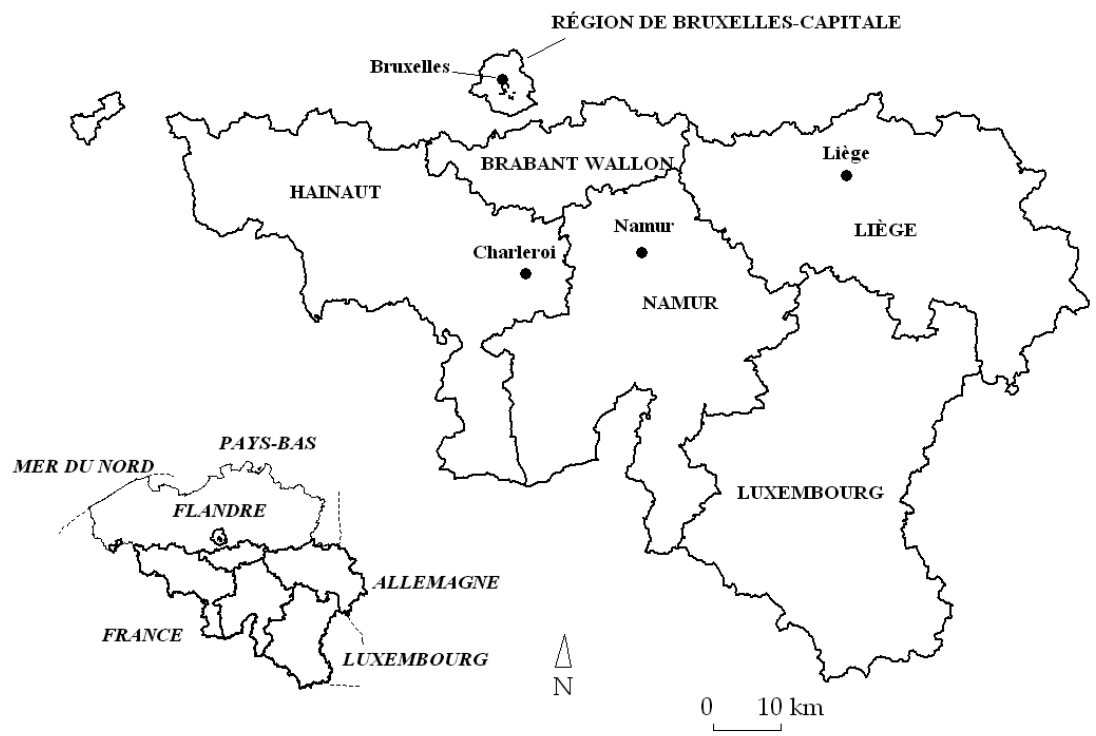

Figure 1 : Carte de localisation de la Wallonie Localization of Wallonia

médian par déclaration, le coût des habitations ou encore le taux de chômage et la proportion de logements disposant d'une connexion Internet.

La dimension environnementale : le milieu rural est classiquement opposé au monde urbain sur la base de critères environnementaux. On a voulu ici combiner une double approche en retenant d'une part, des variables permettant de caractériser un environnement " objectif », sur la base de la qualité de l'air et des superficies consacrées aux bois et à l'agriculture, et d'autre part, un environnement perçu au travers des questions posées lors du recensement de la population et des logements de $2001^{4}$ qui ont permis d'élaborer des indices de satisfaction ${ }^{5}$ relatifs notamment aux espaces verts, à la qualité de l'air, à la tranquillité et à la propreté ${ }^{6}$.

La dimension « service-isolement » : le choix de s'installer ou non à la campagne de même que celui d'y rester sont parfois dictés par des situations d'isolement ou de disponibilité de certains services de proximité (commerce, médecins, administrations, etc.). À défaut de mesures « objectives », nous avons opté pour un isolement "perçu ». Si le résultat est plus subjectif, il n'en est que plus représentatif de la manière dont les individus perçoivent et qualifient leur cadre de vie. Ces informations sont également extraites du recensement de 2001.

Dans la mesure où certaines de ces variables sont étroitement corrélées et afin de s'assurer de leur pertinence, nous avons d'abord procédé à une analyse factorielle en composante principale (avec rotation varimax). Compte tenu de la valeur propre de chaque composante, nous avons retenu 5 facteurs expliquant au total $81,3 \%$ de la variance, soit un score tout à fait satisfaisant (cf. annexe 1).

Ces cinq axes ont des significations complémentaires et distinctes (cf. annexe 2). Le premier est un axe socio-économique, la contribution des variables liées à cette dimension y est la plus forte. Il oppose les communes les plus « riches » aux communes dont les populations connaissent des situations socio-économiques plus difficiles. Le deuxième axe traduit le degré d'isolement.

\footnotetext{
4. Ce recensement fut officiellement dénommé « Enquête socio-économique 2001 ».

5. Ces indices ont été calculés par l'Institut National de Statistique.

6. Ces trois derniers indices ont été synthétisés dans un indice unique appelé « indice global de satisfaction ».
} 


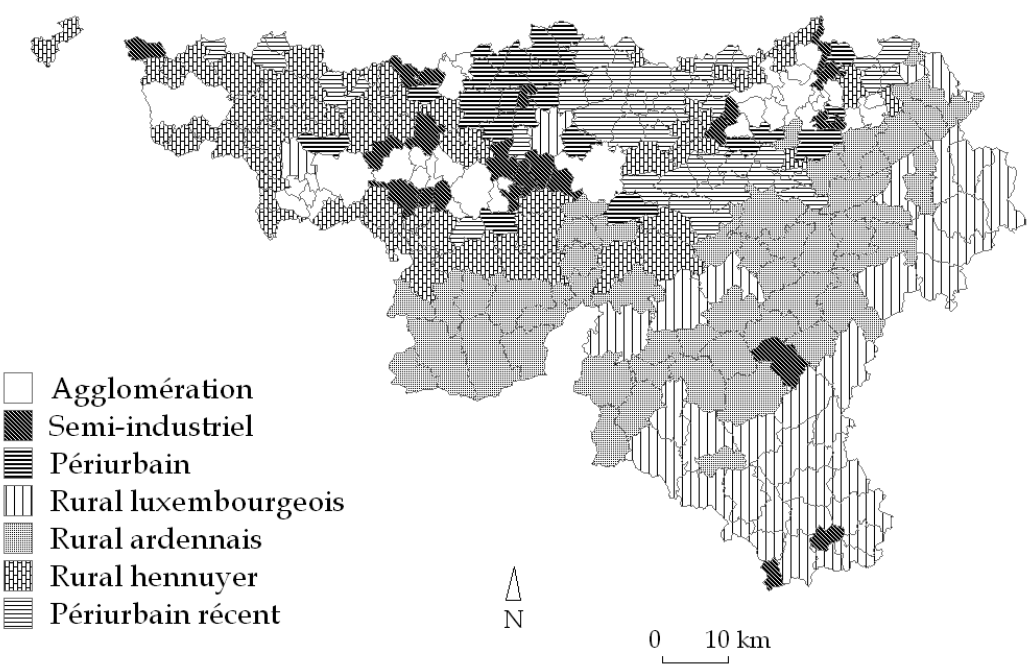

Figure 2 : Identification et localisation des communes wallonnes selon l'analyse de classification Identification and localization of the Walloon communes split among clusters

\begin{tabular}{|c|c|c|}
\hline Dimensions & Variables & Sources \\
\hline Socio-économique & $\begin{array}{l}\text { Revenu médian (2000) } \\
\text { Coût des habitations (2000) } \\
\text { Coût des terrains à bâtir (2000) } \\
\text { Taux de chômage (1998) } \\
\text { Proportion de logement sans chauffage central } \\
\text { (2001) } \\
\text { Proportion de logements disposant d'une } \\
\text { connexion Internet (2001) }\end{array}$ & $\begin{array}{l}\text { (INS, statistiques fiscales) } \\
\text { (INS, statistiques immobilières) } \\
\text { (INS, statistiques immobilières) } \\
\text { (IWEPS) } \\
\text { (INS, Enquête socio-économique) } \\
\text { (INS, Enquête socio-économique) }\end{array}$ \\
\hline Environnement & $\begin{array}{l}\text { Superficie des terres agricoles (2002) } \\
\text { Superficie des terrains boisés (2002) } \\
\text { Émissions acides par km² (1996) } \\
\text { Émissions de particules en suspension par } \mathrm{km}^{2} \\
(1996) \\
\text { Indice de satisfaction global (2001) } \\
\text { Indice de satisfaction quant aux espaces verts } \\
\text { (2001) } \\
\text { Superficie bâtie (2002) }\end{array}$ & $\begin{array}{l}\text { (INS, statistiques cadastrales) } \\
\text { (INS, statistiques cadastrales) } \\
\text { (Atlas de l'air de la Wallonie, 1998) } \\
\text { (Atlas de l'air de la Wallonie, 1998) } \\
\text { (INS, Enquête socio-économique) } \\
\text { (INS, Enquête socio-économique) } \\
\text { (INS, statistiques cadastrales) }\end{array}$ \\
\hline Isolement & $\begin{array}{l}\text { Indice de satisfaction quant à l'offre de services de } \\
\text { santé (2001) } \\
\text { Indice de satisfaction quant à l'offre de } \\
\text { commerces (2001) } \\
\text { Indice de satisfaction quant à l'offre de transport } \\
\text { en commun (2001) } \\
\text { Indice de satisfaction quant à l'offre de services } \\
\text { administratifs (2001) } \\
\text { Indice de satisfaction quant à la présence de } \\
\text { professions libérales (2001) }\end{array}$ & $\begin{array}{l}\text { (INS, Enquête socio-économique) } \\
\text { (INS, Enquête socio-économique) } \\
\text { (INS, Enquête socio-économique) } \\
\text { (INS, Enquête socio-économique) } \\
\text { (INS, Enquête socio-économique) }\end{array}$ \\
\hline
\end{tabular}

Tableau 1 : Liste des variables retenues pour chacune des dimensions Selected covariates within each dimensions 
Il regroupe les variables liées à la satisfaction quant aux services disponibles. Le troisième axe exprime l'intensité de la pollution. Le quatrième axe scinde très clairement le monde rural en deux groupes : les campagnes agricoles et les espaces forestiers. Cette opposition a une forte empreinte spatiale puisqu'elle oppose le sud de la Wallonie, plus boisé (Ardennes), au nord où l'agriculture est davantage développée. Enfin, le cinquième axe exprime l'appréciation du cadre de vie, sur base des indices de satisfaction quant aux espaces verts ou encore par rapport à l'offre de transport.

Sur base de ces axes factoriels, nous avons ensuite réalisé une analyse de classification hiérarchique (méthode de Ward) en deux temps. D'abord, sur l'ensemble des communes retenues, il se dégage cinq groupes : les deux groupes extrêmes sont facilement identifiables, puisqu'il s'agit des profils périurbains et des communes proches des grands centres industriels. Toutefois, ces deux groupes ont des profils tellement marqués que le reste de la Wallonie apparaît comme une masse de communes relativement homogènes. Aussi, nous avons procédé à une seconde classification en excluant les deux groupes précités. À l'issue de ces deux exercices, nous avons combiné l'ensemble des résultats obtenus. Il en résulte un classement de l'ensemble des communes en six classes distinctes (cf. annexe 3 et fig. 2).

Le premier groupe présente un profil semi-industriel. Il s'agit de communes situées à proximité des centres industriels. Il se caractérise par un niveau de pollution élevé et un habitat assez dense. Les habitants sont de condition assez modeste en moyenne et peu satisfaits de leur environnement. Ce groupe inclut les communes de l'ancien sillon industriel Sambre-et-Meuse ainsi que quelques communes du Brabant Wallon et trois communes de la province du Luxembourg.

Le deuxième groupe est de type périurbain. On y retrouve une bonne partie du Brabant Wallon, et quelques communes des périphéries liégeoise et namuroise. Les revenus des habitants y sont en moyenne les plus élevés de même que le coût de l’immobilier. La population est très instruite (en moyenne, $25 \%$ de la population possède un diplôme d'études supérieures contre 13 à $15 \%$ pour les autres groupes). A proximité des grandes agglomérations urbaines et encadrées par des voies de communications rapides, la qualité de l'air n'y est pas la plus favorable. Par contre, les indices de satisfaction par rapport à l'environnement et par rapport à l'offre de services de santé sont très positifs.

Le troisième groupe se caractérise par une forte proportion de zones boisées (41\%). Les communes sont très peu polluées, les indicateurs sociaux sont peu favorables - taux de chômage élevé et revenu médian très faible -, les habitants sont mécontents des services offerts tandis que leur point de vue sur l'environnement est très positif. Ce groupe qui englobe l'espace frontalier avec l'Allemagne est essentiellement centré sur le sud de la province du Luxembourg. Nous l'appellerons le type « rural luxembourgeois »

Le quatrième groupe reprend une bonne part des caractéristiques du groupe précédent. Les superficies boisées y sont les plus importantes et les indices de satisfaction par rapport à l'environnement y sont les plus élevés. Sur la carte, ces communes constituent une sorte de zone «tampon » entre le sud et les autres communes wallonnes. Cette bande intermédiaire couvre grosso modo les Ardennes et reprend les zones de loisirs des vallées de la Lesse et de l'Ourthe-Amblève. Ce groupe sera dénommé « rural ardennais».

Le groupe 5, appelé « rural hennuyer », comprend des communes caractérisées par des superficies agricoles importantes (70\% en moyenne). Pour ce qui est des variables socio-économiques, si le revenu médian est assez faible, le taux de chômage y est élevé et les logements mal équipés (que ce soit en matière de chauffage central ou de connexion Internet). Il s'agit donc d'une population assez modeste, peu instruite et relativement peu satisfaite quant à l'environnement dans lequel elle vit.

Enfin, le groupe 6 correspond au nouveau périurbain ou périurbain récent. Ces communes constituent la zone d'extension récente des migrations périurbaines (principalement au départ de Bruxelles). Ces communes ont un profil très proche des communes périurbaines plus anciennes, tout en étant un peu moins riches, moins polluées et légèrement moins bien considérées sur un plan environnemental. 


\section{Diversité des évolutions, des caractéristiques et des comportements démographiques}

Ces six groupes ont été construits sans prendre en compte de variables démographiques. Ce choix était délibéré puisqu'il s'agit de vérifier ensuite si la diversité des espaces ruraux délimités se traduit ou non par des caractéristiques et des comportements démographiques spécifiques.

\section{L'ÉVOLUTION DE LA POPULATION DES DIFFÉRENTS GROUPES DE 1978 À 2002}
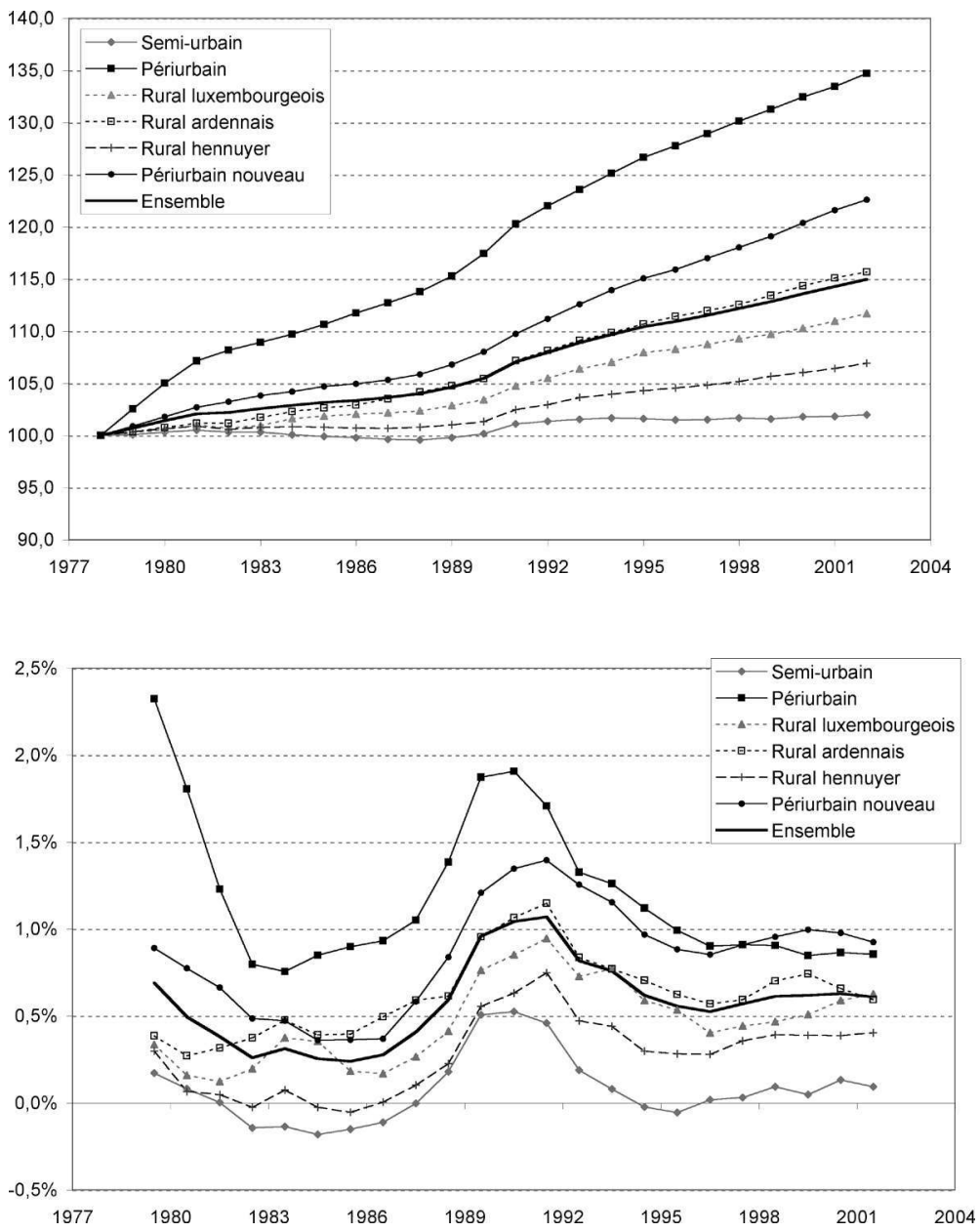

Figure 3 : L'évolution de la population des 6 groupes de communes de 1978 à $2002(1978=100)$ (Source : Registre national-Institut National de Statistique) Demographic evolution of the 6 commune clusters from 1978 to $2002(1978=100)$
Figure 4 : Taux d'accroissement annuel (moyenne mobile sur trois ans) Source : Registre nationalInstitut National de Statistique

rate (3 Anmual growt Mean)

De $1978^{7}$ à 2002, le groupe périurbain voit sa population augmenter de plus de $35 \%$ (fig. 3). C'est la croissance la plus forte et la tendance qui se dessine dès 1979 montre clairement que le processus est bien antérieur à cette date. Le nouveau périurbain connaît une faible croissance de

7. Suite à la fusion des communes réalisée en 1977 (le nombre de communes fut ramené d’environ 2600 à 589 ), 1978 est la première année pour laquelle on dispose de séries homogènes de chiffres de population sur base du nouveau découpage communal. 
sa population jusqu'au début des années 1990, puis une nette accélération se dessine. À partir de 1997, le taux d'accroissement du nouveau périurbain dépasse celui du périurbain (fig. 4), ce qui pourrait révéler un effet de saturation de la zone périurbaine qui « déborde » vers les communes adjacentes.

À l'opposé de ces deux groupes marqués par une forte croissance démographique, les communes semi-industrielles et le rural hennuyer enregistrent des croissances quasi nulles (dans les années 1980 on observe même des taux d'accroissement négatifs). Tout indique que dans le groupe semi-industriel, ne restent que les personnes qui n'ont pas d'autres choix. Pour le rural hennuyer, la situation est un peu différente et on peut supposer qu'il bénéficie (surtout à partir des années 1990) de l'effet de saturation qui marque les communes périurbaines. Face à la hausse des loyers et du coût de l'immobilier dans ces dernières, une partie de leurs habitants de même que certains ménages qui, a priori, auraient plutôt opté pour la zone périurbaine, sont obligés de s’installer plus loin, dans des communes moins favorisées, mais également moins touchées par les hausses de prix. Les ménages qui gagnent ainsi le rural hennuyer n'ont probablement pas le même profil socio-économique que ceux qui s’installent dans le périurbain récent (Hermia et al., 2005). La valeur du foncier et de l'immobilier détermine dans une large mesure le profil socio-économique des nouveaux arrivants.

Entre ces deux extrêmes, on retrouve le rural luxembourgeois et le rural ardennais. Ce dernier voit sa population augmenter tout au long de la période. Elle croît plus vite en moyenne que celle du rural luxembourgeois, même si une inversion de tendance se manifeste depuis la fin des années 1990. On peut y voir les effets de la périurbanisation de la ville de Luxembourg (Grand-Duché) qui progressivement se développe dans l'extrême sud de la Wallonie (Eggerickx et al., 2002a).

En résumé, abstraction faite des soubresauts conjoncturels, ces types de communes rurales se caractérisent au cours de la dernière décennie par une croissance de leur population, mais à des rythmes bien différents. À ce niveau, les campagnes wallonnes participent au processus de renouveau démographique qui caractérise de nombreux espaces ruraux en Europe occidentale (Datar, 2003, Luginbühl, 2007). Au-delà des simples chiffres d'évolution de la population, les agents sociodémographiques de cette croissance et les populations résidant dans ces différents types ruraux présentent bien des différences.

\section{DES CARACTÉRISTIQUES ET DES COMPORTEMENTS DÉMOGRAPHIQUES DIFFÉRENTS}

Les contrastes socio-économiques et environnementaux qui caractérisent ces milieux « ruraux » se traduisent par des différences de caractéristiques et de comportements démographiques. Le tableau 2 présente quelques indicateurs de fécondité, de mortalité, de structure par âge et de type de ménage.

Le milieu périurbain se détache nettement avec des indices de mortalité nettement plus favorables que dans les autres groupes. L'espérance de vie à la naissance - qui illustre le mieux les conditions de vie d'une population - y est de 78,2 ans, pour à peine plus de 76 ans dans les communes semi-industrielles et le rural hennuyer. Les différences marquent essentiellement la mortalité masculine, notamment aux âges élevés. Entre 50 et 74 ans, un homme résidant en milieu périurbain a un risque de mourir de 20 à $40 \%$ moins élevé que s'il résidait en milieu semi-industriel ou dans le rural hennuyer. On observe que le périurbain récent se caractérise également par des indices de mortalité plus favorables que les autres zones rurales. Le niveau de vie des populations, l'accès différencié aux soins de santé, les comportements liés à la consommation de tabac, d'alcool ou en matière de prévention médicale expliquent généralement ces différences.

L'homogénéisation des indices de (basse) fécondité est un processus bien connu. Il n'empêche que si l'indice synthétique de fécondité présente peu de différence, il en est autrement de son calendrier. Ici aussi, le périurbain se démarque avec une fécondité plus tardive, comme en témoigne les indices de fécondité réalisée entre 30 et 49 ans : 86,4\%o pour 64,1\%o dans les communes semi-industrielles. Cette particularité résulte notamment d'un report de la maternité 
Thierry Eggerickx, Jean-Paul Sanderson, Amel Bahri, Jean-Pierre Hermia

\begin{tabular}{|c|c|c|c|c|c|c|c|}
\hline Indices & $\begin{array}{l}\text { Semi- } \\
\text { industriel }\end{array}$ & Périurbain & $\begin{array}{c}\text { Rural } \\
\text { Luxemb. }\end{array}$ & $\begin{array}{c}\text { Rural } \\
\text { ardennais }\end{array}$ & $\begin{array}{c}\text { Rural } \\
\text { hennuyer }\end{array}$ & $\begin{array}{c}\text { Périurbain } \\
\text { nouveau }\end{array}$ & Ensemble \\
\hline $\begin{array}{l}\text { ICFécondité (1997-2002) } \\
\text { (enfant(s) par femme) }\end{array}$ & 1,69 & 1,70 & 1,80 & 1,79 & 1,71 & 1,74 & 1,74 \\
\hline $\begin{array}{l}\text { Part de fécondité réalisée } \\
\text { entre 30-49 ans (1997- } \\
\text { 2002) }\end{array}$ & $64,1 \%$ & $86,4 \%$ & $73,1 \%$ & $70,6 \%$ & $67,4 \%$ & $74,9 \%$ & $65,8 \%$ \\
\hline $\begin{array}{l}\text { Espérance de vie à la } \\
\text { naissance ( } 2 \text { sexes) (1997- } \\
\text { 2002) }\end{array}$ & 76,5 ans & 78,2 ans & 76,9 ans & 76,6 ans & 76,2 ans & 77,0 ans & 76,7 ans \\
\hline $\begin{array}{l}\text { Espérance de vie à la } \\
\text { naissance (hommes) (1997- } \\
2002 \text { ) }\end{array}$ & 72,8 ans & 75,3 ans & 73,2 ans & 73,1 ans & 72,6 ans & 73,6 ans & 73,3 ans \\
\hline $\begin{array}{l}\text { Quotient de mortalité entre } \\
50-74 \text { ans (ho.) (1997-2002) }\end{array}$ & $87,9 \%$ & $67,9 \%$ & $79,3 \%$ & $80,9 \%$ & $89,1 \%$ & $77,3 \%$ & $82,3 \%$ \\
\hline Âge moyen (1-1-2002) & 39,5 ans & 38,4 ans & 38,6 ans & 39,5 ans & 39,5 ans & 38,9 ans & 39,1 ans \\
\hline$<20$ ans $(1-1-2002)$ & $24,7 \%$ & $26,2 \%$ & $26,1 \%$ & $25,5 \%$ & $24,7 \%$ & $25,8 \%$ & $25,4 \%$ \\
\hline 60 ans et $+(1-1-2002)$ & $21,1 \%$ & $18,7 \%$ & $20,6 \%$ & $21,9 \%$ & $20,9 \%$ & $20,2 \%$ & $20,7 \%$ \\
\hline Isolés (1-1-2002) & $27,2 \%$ & $25,2 \%$ & $26,8 \%$ & $29,2 \%$ & $27,3 \%$ & $24,2 \%$ & $26,9 \%$ \\
\hline $\begin{array}{l}\text { Couples mariés avec } \\
\text { enfant(s) (1-1-2002) }\end{array}$ & $27,8 \%$ & $31,6 \%$ & $32,1 \%$ & $28,5 \%$ & $27,9 \%$ & $31,5 \%$ & $29,5 \%$ \\
\hline $\begin{array}{l}\text { Couples mariés sans enfant } \\
(1-1-2002)\end{array}$ & $15,8 \%$ & $14,3 \%$ & $19,9 \%$ & $20,1 \%$ & $20,0 \%$ & $20,4 \%$ & $20,1 \%$ \\
\hline Monoparentaux (1-1-2002) & $10,6 \%$ & $9,6 \%$ & $9,2 \%$ & $9,4 \%$ & $10,2 \%$ & $9,3 \%$ & $9,8 \%$ \\
\hline Cohabitants (1-1-2002) & $8,8 \%$ & $9,3 \%$ & $7,7 \%$ & $8,9 \%$ & $9,7 \%$ & $9,2 \%$ & $9,0 \%$ \\
\hline $\begin{array}{l}\text { Ménage de } 5 \text { personnes et } \\
\text { plus (1-1-2002) }\end{array}$ & $7,6 \%$ & $9,9 \%$ & $9,5 \%$ & $8,6 \%$ & $7,8 \%$ & $9,3 \%$ & $8,6 \%$ \\
\hline
\end{tabular}

Tableau 2 : Quelques indicateurs démographiques selon le milieu rural de résidence Source : Registre national et Institut National de Statistique.

\section{Some demographic indicators according to rural place of residence}

après les études. Le périurbain est le type qui se caractérise par la plus forte proportion d’individus possédant un niveau d'instruction élevé (Eggerickx et al., 2002b). On observe alors un effet de rattrapage de la fécondité aux âges plus avancés. Inversement, dans les zones semi-industrielles et dans le rural hennuyer, le calendrier de la fécondité est très précoce : dans ces deux groupes, $60 \%$ de la fécondité des femmes est déjà réalisé avant 30 ans, pour moins de $50 \%$ dans les communes périurbaines. Cette précocité du calendrier de la fécondité se retrouve également dans les régions rurales ardennaise et luxembourgeoise, mais associée cette fois à une fécondité plus élevée. Il s'agit là d'une tradition ancienne, marquée par une transition de la fécondité plus tardive et plus lente que dans les autres régions de Wallonie (Rapport Poliwa, 1977). Aujourd'hui encore, ces régions ardennaises se caractérisent par les niveaux de fécondité les plus élevés de Belgique, signes d'un certain conservatisme des comportements.

Le milieu périurbain se démarque également en termes de vieillissement démographique : l'âge moyen de la population y est le plus bas, la part relative des moins de 20 ans la plus élevée et la proportion de personnes âgées de plus de 60 ans la plus faible. À l'opposé, les indices de 
vieillissement sont les plus marqués dans le milieu semi-industriel, le rural hennuyer et le rural ardennais. Ce dernier se distingue d'ailleurs par la part relative de seniors la plus élevée. Enfin, autre trait majeur : la proportion de moins de 20 ans est élevée dans le rural luxembourgeois - conséquence d'une fécondité-natalité plus importante - alors que la part relative des aînés est également importante.

La distribution selon le type de ménage présente également des différences significatives selon le milieu rural de résidence.

Les couples mariés avec enfant(s) restent, dans ces milieux ruraux, le type de ménage le mieux représenté. C'est principalement le cas des milieux périurbains où ils constituent l'une des principales composantes démographiques des nouveaux arrivants. On en trouve également une proportion élevée dans le milieu rural luxembourgeois. Rappelons que cette région, à proximité d'Arlon, bénéficie d'une forte exurbanisation de la ville de Luxembourg avec un double mouvement : d'une part de jeunes couples belges avec enfant(s) qui s'installent dans cette région et travaillent au Grand Duché de Luxembourg, et d'autre part, des Luxembourgeois, qui viennent résider de l'autre côté de la frontière, là où les prix des maisons et des terrains sont encore relativement abordables.

Les ménages de personnes isolées sont très présents, et talonnent en termes de poids relatif les couples mariés avec enfant(s), dans les régions semi-industrielles, dans le rural hennuyer et surtout dans le rural ardennais.

Les ménages monoparentaux et de cohabitants ${ }^{8}$ sont les moins bien représentés dans le rural luxembourgeois, ce qui tend à souligner une fois de plus les comportements plus traditionnels qui caractérisent cet espace. Inversement, la part relative des monoparentaux est la plus élevée dans les régions plus pauvres semi-industrielles et du rural hennuyer.

Enfin, les couples mariés sans enfant, un type de ménage généralement âgé (âge moyen supérieur à 60 ans), sont les moins bien représentés dans les milieux périurbain et semi-industriel.

Les différents types de ménage ne partagent donc pas forcément les mêmes types d'espaces ruraux. Comme l'indiquent les corrélations présentées au tableau 3, il existe une opposition spatiale marquée entre, d'une part, les couples mariés avec enfants, et d'autre part, les ménages monoparentaux et d'isolés. Cette spatialisation des types de ménage se double d'un marquage social. Ainsi, la distribution spatiale des ménages monoparentaux féminins est significativement corrélée à celle du taux de chômage $(\mathrm{r}=+0,72)$ et à la part relative de faibles revenus $(\mathrm{r}=+0,51)$. Inversement, il y a une association négative entre la distribution des couples mariés avec enfants et le taux de chômage $(r=-0,72)$, ainsi qu'avec la proportion de faibles revenus $(r=-0,68)$.

Les différences observées dans la répartition par âge de la population, dans la distribution des types de ménages, voire dans les comportements de fécondité et de mortalité, sont plus ou moins étroitement déterminées par le profil démographique et social de la migration.

\section{Les comportements migratoires : le rôle déterminant de l'âge}

À l'échelle locale, en Wallonie comme pour de nombreuses autres régions d'Europe (Champion, 2000 ; Baccaïni, 2000, Perrier-Cornet, 2003), les migrations constituent le principal moteur de l'évolution démographique. Schématiquement, en Wallonie, les milieux urbains, et principalement les grandes villes, affichent des bilans migratoires ${ }^{9}$ négatifs, alors que les autres communes bénéficient généralement d'une attractivité migratoire. C'est le cas des six groupes qui se caractérisent tous par des bilans migratoires positifs. L'intensité de ces derniers est toutefois très variable. Au cours de la période quinquennale 1997-2002, elle varie de $+0,1 \%$ dans le milieu semi-industriel

8. Il s'agit de personnes vivant ensemble mais non mariées.

9. Il s'agit de la différence entre les immigrations et les émigrations. 
Thierry Eggerickx, Jean-Paul Sanderson, Amel Bahri, Jean-Pierre Hermia

\begin{tabular}{|l|c|c|c|c|}
\hline Indices & CAE & MF & IH & IF \\
\cline { 1 - 4 } Couples mariés avec enfants (CAE) & & $-0,64$ & $-0,65$ & $-0,76$ \\
Monoparentaux féminins (MF) & $-0,64$ & & 0,20 & 0,43 \\
Isolés Hommes (IH) & $-0,65$ & 0,20 & & 0,62 \\
Isolés Femmes (IF) & $-0,76$ & 0,43 & 0,62 & \\
\cline { 1 - 1 } & & & & 0,53 \\
\cline { 1 - 1 } Taux de Chômage & $-0,72$ & 0,72 & 0,45 & $-0,58$ \\
Revenu médian & 0,58 & $-0,40$ & $-0,60$ & 0,55 \\
\hline
\end{tabular}

Tableau 3 : Matrice de corrélation linéaire (r) entre types de ménage et indicateurs socio-économiques (Source : Registre national et Institut National de Statistique)

Correlation matrix between household types proportions and socioeconomic indicators

à $+4,0 \%$ dans le périurbain nouveau. Il s'agit là des communes les plus attractives, suivies du groupe périurbain et du rural ardennais.

Ces migrations correspondent à des tranches d'âges, à des types de ménages et à des catégories socioprofessionnelles spécifiques (Van Hecke et Savenberg, 2002). Autrement dit, les mouvements migratoires ont une influence déterminante, non seulement sur l'évolution, à la hausse ou à la baisse, du chiffre de la population d'une commune, mais également sur ses caractéristiques démographiques, socio-économiques et culturelles. Des courants migratoires plus importants et plus spécifiques à certains groupes d'âges sont observés vers des lieux bien déterminés en réponse à des attraits bien précis. Il en résulte inévitablement une recomposition plus ou moins marquée de la structure par âge ; recomposition qui peut favoriser un rajeunissement de la pyramide, si la commune est attractive pour les jeunes ménages et leurs enfants, ou, au contraire, en accélérer le vieillissement, si elle attire les personnes âgées et/ou repousse les jeunes adultes (Eggerickx et al., 2002b). L'examen des soldes migratoires par groupe d'âges (figure 5 et tableau 4) illustre parfaitement notre propos.

\section{ÉTALEMENT DE LA PERIURBANISATION \\ ET BLOCAGE SOCIO-DÉMOGRAPHIQUE DE L'ESPACE PÉRIURBAIN}

Les zones périurbaines et périurbaines récentes présentent un calendrier de la migration par âge typique : une attractivité migratoire très élevée d'adultes âgés de 30-39 ans et de leur(s) enfant(s) de moins de 10 ans. Cette tendance est davantage marquée dans le groupe des communes de périurbanisation récente qui, outre des bilans migratoires plus importants pour les trentenaires, se distingue aussi par des bilans positifs pour les 25-29 ans. En d'autres termes, pour ce groupe de communes, le calendrier de la migration est plus jeune que celui des communes périurbaines, d'autant que pour ces dernières, le bilan migratoire des 25-29 ans est très négatif.

Ce double constat semble confirmer le verrouillage socio-démographique qui touche ces communes périurbaines. Le mécanisme est le suivant (Eggerickx et al., 2002b). Cette zone périurbaine, centrée pour l'essentiel sur le Brabant Wallon, à proximité de l'agglomération de Bruxelles, bénéficie d'une croissance démographique rapide depuis le début des années 1950. La population du Brabant Wallon a doublé, et depuis 1970, cette zone assure environ deux tiers de la croissance démographique régionale (Hermia et al., 2005). La proximité de Bruxelles, un réseau de voies de communication rapides, un cadre environnemental plaisant expliquent cet essor démographique, qui n'est pas sans revers. Cette attractivité migratoire s'est accompagnée d'une hausse très rapide du prix de l'immobilier et du foncier. Ainsi, en 2000, le prix moyen d'une maison y était de 105000 euros pour 64000 euros (tableau 5) dans la zone semi-industrielle ou 84000 euros 

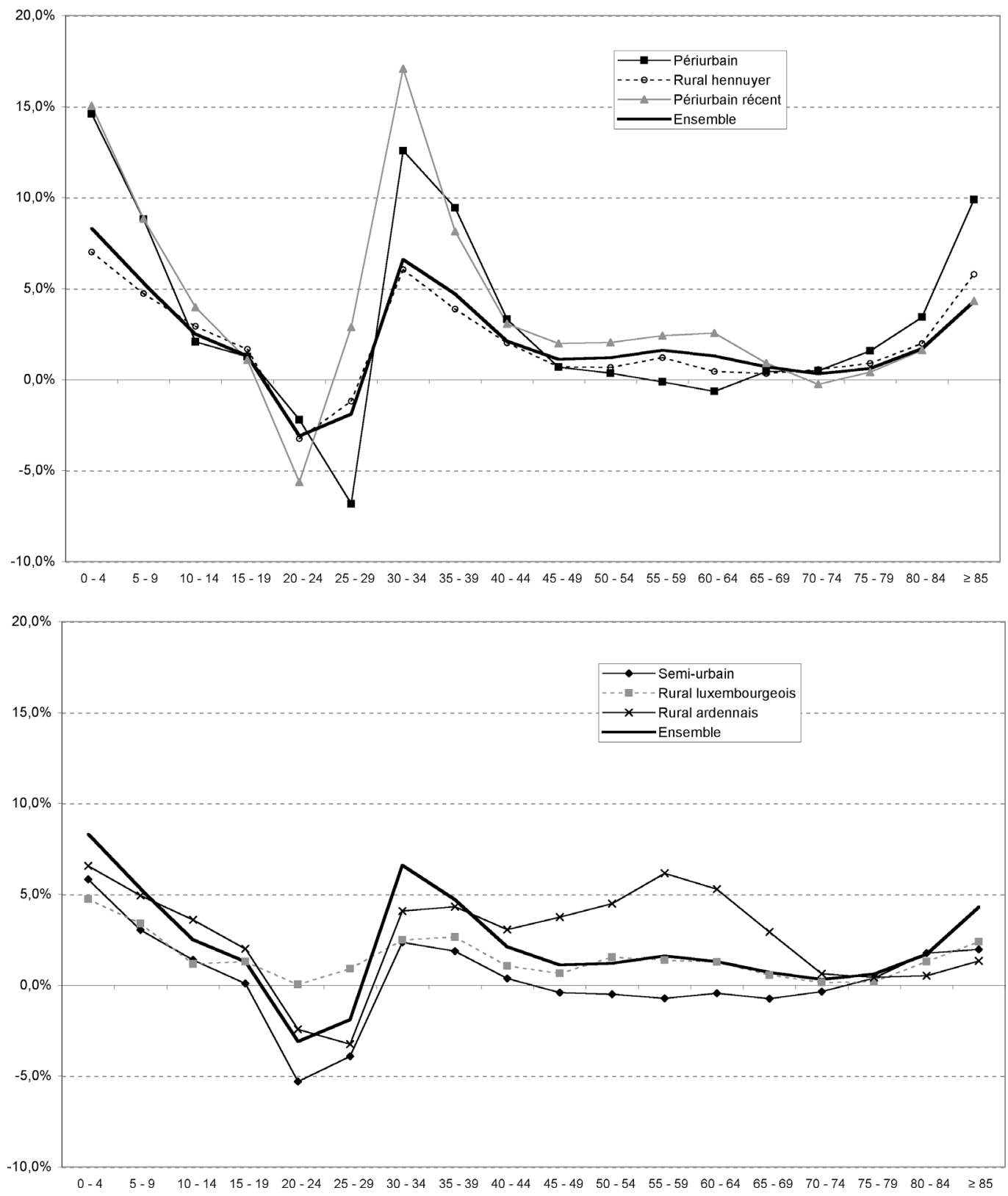

Figure 5 : Les bilans migratoires par groupe quinquennal d'âges selon les groupes de communes (1997-2002) (Source : Registre national-Institut National de Statistique)

Migratory balances for 5-year age groups by commune clusters (1997-2002)

dans le périurbain récent. Le prix des terrains à bâtir était deux fois plus élevé dans le périurbain que dans le périurbain nouveau et le rural hennuyer. Un processus de sélection sociale par l'immigration est donc à l'œuvre dans ces communes périurbaines : seuls les ménages disposant de revenus confortables ont encore la possibilité de se payer un logement décent dans ces communes (De Bie, 1996). Ces mécanismes de spécialisation sociodémographique des espaces ne sont pas 


\begin{tabular}{|l|c|c|c|c|c|c|c|}
\hline Indices & $\begin{array}{c}\text { Semi- } \\
\text { industriel }\end{array}$ & Périurbain & $\begin{array}{c}\text { Rural } \\
\text { luxembour- } \\
\text { geois }\end{array}$ & $\begin{array}{c}\text { Rural } \\
\text { ardennais }\end{array}$ & $\begin{array}{c}\text { Rural } \\
\text { hennuyer }\end{array}$ & $\begin{array}{c}\text { Périurbain } \\
\text { nouveau }\end{array}$ & Ensemble \\
\hline BM Total & $0,1 \%$ & $2,9 \%$ & $1,5 \%$ & $2,8 \%$ & $1,8 \%$ & $4,0 \%$ & $2,0 \%$ \\
BM <15 ans & $3,3 \%$ & $8,1 \%$ & $3,0 \%$ & $4,9 \%$ & $4,8 \%$ & $9,0 \%$ & $5,7 \%$ \\
BM 15-24 ans & $-2,6 \%$ & $-0,5 \%$ & $0,7 \%$ & $-0,1 \%$ & $-0,8 \%$ & $-2,1 \%$ & $-1,6 \%$ \\
BM 30-44 ans & $1,5 \%$ & $8,1 \%$ & $2,1 \%$ & $3,8 \%$ & $3,9 \%$ & $9,0 \%$ & $5,0 \%$ \\
BM 55-69 ans & $-0,7 \%$ & $-0,1 \%$ & $1,1 \%$ & $4,8 \%$ & $0,7 \%$ & $2,0 \%$ & $1,3 \%$ \\
\hline Tx mobilité & $51,0 \%$ & $69,8 \%$ & $50,5 \%$ & $56,6 \%$ & $52,7 \%$ & $56,0 \%$ & $55,3 \%$ \\
\hline
\end{tabular}

Tableau 4 : Les bilans migratoires par grand groupe d'âges (1997-2002) (Source : Registre national et Institut National de Statistique)

Migratory balances for large age groups (1997-2002)

\begin{tabular}{|l|c|c|c|c|c|c|}
\hline \multirow{2}{*}{ Types de communes rurales } & \multicolumn{2}{|c|}{$\begin{array}{c}\text { Maisons d'habitation ordinaire } \\
\text { prix moyen en euro par bâtiment) }\end{array}$} & \multicolumn{3}{|c|}{$\begin{array}{c}\text { Terrains à bâtir } \\
\text { (prix moyen en euro par } \mathrm{m}^{2} \text { ) }\end{array}$} \\
\cline { 2 - 7 } & 1995 & 2000 & Évolution & 1995 & 2000 & Évolution \\
\hline Semi-industriel & 50.614 & 64.071 & $+27 \%$ & 23,2 & 31,9 & $+37 \%$ \\
Périurbain & 86.214 & 105.003 & $+22 \%$ & 38,2 & 58,3 & $+53 \%$ \\
Rural luxembourgeois & 60.743 & 76.503 & $+26 \%$ & 18,9 & 22,3 & $+18 \%$ \\
Rural ardennais & 57.763 & 75.038 & $+30 \%$ & 15,0 & 20,1 & $+34 \%$ \\
Rural hennuyer & 51.458 & 68.500 & $+33 \%$ & 19,4 & 28,5 & $+47 \%$ \\
Périurbain récent & 63.061 & 84.253 & $+34 \%$ & 19,6 & 28,7 & $+46 \%$ \\
Ensemble & 61.642 & 78.895 & $+28 \%$ & 22,4 & 31,6 & $+41 \%$ \\
\hline
\end{tabular}

Tableau 5 : L'évolution du coût des maisons et des terrains à bâtir (Source : Institut National de Statistique, Statistiques immobilières)

\section{Price evolution for house and building land}

propres à la Wallonie et s'observent dans de nombreux pays européens (notamment) (Arbonville, 2006; Maurin, 2004).

Ce verrouillage social affecte en particulier les jeunes ménages en phase de décohabitation et/ou ceux qui disposent de revenus moyens et qui souhaitent accéder à la propriété, contraints de prospecter plus loin (notamment par rapport à Bruxelles), vers des régions plus isolées où leurs aspirations résidentielles (la maison quatre façades avec un jardin) peuvent encore être concrétisées. Une étude récente ${ }^{10}$, basée sur l'analyse des matrices origine-destination des émigrants entre 1991-1995 et 2001-2005, a démontré une augmentation très sensible de la propension à émigrer de la zone périurbaine vers les communes environnantes (périurbain récent) des jeunes ménages. Entre les deux périodes quinquennales, ce taux d'émigration s'est accru de $39 \%$ pour les ménages dont les conjoints sont âgés de 20-24 ans et de $73 \%$ pour ceux qui ont entre 25 et 29 ans.

Ceci explique l'extension de la périurbanisation à l'est du Brabant Wallon (groupe périurbain récent), et dans une moindre mesure, vers l'ouest, vers les communes rurales hennuyères. Ces communes affichent également un calendrier migratoire de type périurbain, même si l'intensité des bilans migratoires entre 30 et 39 ans et aux jeunes âges est moins marquée. Cet attrait migratoire se traduit par une augmentation rapide du prix des maisons et des terrains à bâtir (tableau 5).

10. Sanderson (J.-P.), EggerickX (T.), Bahri (A.), 2007. - « Transformation des ménages et ségrégation sociale de l'espace. Une analyse de la précarisation des parcours résidentiels en Belgique (1991-2005) ", Chaire Quetelet 2007, Dynamiques de pawvreté et vulnérabilités, Institut de Démographie, UCL, Louvain-la-Neuve (à paraître). 
En effet, selon les données immobilières fournies par l'Institut National de Statistique, ces deux groupes de communes (nouveau périurbain et rural hennuyer) ont connu une augmentation de $33 \%$ du prix des maisons entre 1995 et 2000, contre $22 \%$ dans les communes périurbaines. Si ces dernières se caractérisent par la plus forte augmentation $(+53 \%)$ du prix des terrains à bâtir, elles sont suivies de près par les communes du rural hennuyer et du périurbain nouveau, avec respectivement une croissance de 47 et de $46 \%^{11}$.

On assiste donc à un étalement de plus en plus diffus de la périurbanisation qui, outre ses conséquences sociodémographiques, modifie également les équipements, les paysages, l'environnement, l'habitat, l'économie, les rapports sociaux et les modes de vie du monde rural.

\section{LA MIGRATION DE PERSONNES ÂGÉES : RETRAITE ET VEUVAGE}

L'arrivée à la retraite constitue une autre période-clé dans le calendrier de la migration. Traditionnellement, les milieux urbanisés en Wallonie enregistrent des bilans migratoires négatifs chez les futurs et/ou jeunes retraités. Les communes semi-industrielles prolongent cette tendance (fig. 5). L'autre groupe pour lequel on observe une répulsivité des personnes âgées de 55-64 ans est celui des communes périurbaines plus anciennes, alors que le périurbain récent se révèle très attractif pour cette tranche d'âges. Celui-ci semble récupérer, au moins en partie, cette population en provenance de communes qui se sont sensiblement urbanisées (densité de population et d'habitat de plus en plus élevée) et dont la qualité environnementale s’est modifiée, voire détériorée, au cours des dernières décennies.

Le calendrier de la migration des communes du rural ardennais est tout à fait particulier. Ces communes sont à la fois attractives pour les adultes de 30-44 ans et leur(s) enfant(s) et pour les personnes de 50-69 ans. Le groupe modal se situe entre 55 et 64 ans, ce qui témoigne de la forte attractivité exercée par ces communes sur les jeunes retraités. Rappelons que ce groupe de communes se caractérise par les proportions de personnes âgées de plus de 60 ans, d'isolés et de couples sans enfants les plus élevées (tableau 2).

Enfin, tous les groupes de communes affichent des bilans migratoires positifs pour les personnes âgées de plus de 80 ans. Cette tendance est particulièrement marquée dans le groupe périurbain où, en termes relatifs, le bilan migratoire des personnes âgées de plus de 85 ans est même supérieur à celui des 35-39 ans. Cette attractivité migratoire, bien souvent liée au veuvage, s'explique en partie par la présence de nombreuses institutions d'accueil pour personnes âgées et par la localisation spatiale de ces communes, à proximité des grands centres urbains et des voies de communication rapides. Rappelons que ces communes se caractérisent par les indices de satisfaction les plus élevés quant à la proximité de services de soins de santé.

\section{L'IMPACT DE LA MIGRATION SUR L'ÉVOLUTION DU VIEILLISSEMENT DÉMOGRAPHIQUE}

L'impact des mouvements migratoires sur la structure par âge est évident. Les sortants sont remplacés par des entrants, mais les uns et les autres ne sont semblables, ni quant à leur effectif, ni quant à leur âge moyen. Une méthode a donc été mise au point afin de déterminer l'impact des différentes composantes du mouvement de la population ${ }^{12}$ - les migrations internes, les migrations internationales et le bilan naturel - sur l'évolution de l'âge moyen de la population des communes belges au cours de la période 1992-2002. L'intérêt de la méthode (cf. annexe 4) est de mettre en évidence le rôle particulier joué par chacune de ces composantes démographiques

11. À titre de comparaison, cette augmentation des terrains à bâtir fut de $37 \%$ dans les communes semi-industrielles, de $34 \%$ dans les communes rurales ardennaises et de $18 \%$ dans le rural luxembourgeois.

12. Cette méthode a fait l'objet d'une communication : Sanderson (J.-P.), EgGerickX (T.), Poulain (M.), 2004. - « Impact des migrations internes sur le vieillissement des populations locales : les communes belges de 1991 à 2001 », dans Reproduction et renouvellement des populations, XIII e Colloque National de Démographie, Conférence Universitaire de Démographie et d'Études des Populations (CUDEP), 11-14 mai 2004, Dijon, (à paraître). 


\begin{tabular}{|l|c|c|c|}
\hline \multicolumn{1}{|c|}{$\begin{array}{c}\text { Groupe de communes } \\
\text { rurales }\end{array}$} & Impact du bilan naturel & $\begin{array}{c}\text { Impact des migrations } \\
\text { internes }\end{array}$ & $\begin{array}{c}\text { Impact des migrations } \\
\text { internationales }\end{array}$ \\
\hline Semi-industriel & $-8,22$ ans & $-0,04$ an & $-0,10$ an \\
Périurbain & $-7,64$ ans & $-0,88$ an & $-0,21$ an \\
Rural luxembourgeois & $-8,56$ ans & $-0,45$ an & $-0,05$ an \\
Rural ardennais & $-8,70$ ans & $-0,36$ an & $-0,07$ an \\
Rural hennuyer & $-8,54$ ans & $-0,51$ an & $-0,08$ an \\
Périurbain récent & $-8,55$ ans & $-1,23$ an & $-0,07$ an \\
Ensemble & $-8,40$ ans & $-0,54$ an & $-0,09$ an \\
\hline \multicolumn{3}{|r|}{} \\
\hline Agglomérations urbaines & $-8,56$ ans & $+0,23$ an & $-0,28$ an \\
\hline Total Wallonie & $-8,47$ ans & $-0,23$ an & $-0,17$ an \\
\hline
\end{tabular}

Tableau 6 : L'impact des composantes du mouvement de la population sur l'évolution de l'âge moyen des communes rurales de Wallonie, entre 1992 et 2002

in Wallonia (1992-2002)

Impact of the components from demographic movements on mean age evolution in rural communes

dans le vieillissement des populations locales. Sans migration ni mouvement naturel, la population aurait vieilli de 10 ans entre 1992 et 2002 : il s'agit du vieillissement « synchronisé ». Pourtant, au cours de cette période, l'âge moyen des communes périurbaines (par exemple) est passé de 36,6 ans à 38,4 ans soit un peu moins de deux ans : il s'agit du vieillissement « observé ». La différence entre les vieillissements synchronisé et observé est «absorbée » par les migrations et le mouvement naturel.

Comme le montre le tableau 6, l'impact du mouvement naturel explique l'essentiel des différences observées entre le vieillissement synchronisé et le vieillissement observé. Toutefois, le rôle des migrations internes n'est pas négligeable. Dans les agglomérations urbaines (exclues de la présente étude), les migrations internes, marquées par le départ des jeunes couples avec enfant(s) notamment, accentuent le vieillissement démographique. À l'inverse, dans tous les groupes ruraux considérés, les migrations internes contribuent au rajeunissement relatif de la structure par âge de la population.

L'impact des migrations internes est cependant variable. Il est quasiment nul dans les communes semi-industrielles, qui se rapprochent du cas des agglomérations urbaines. Il est par contre nettement plus significatif dans le périurbain et le périurbain récent, grâce à l'arrivée de nombreux jeunes ménages avec enfant(s). Si on calcule les corrélations entre la valeur de l'impact des migrations internes et les bilans migratoires par groupes d'âges, la seule relation significative concerne les 30-44 ans (coefficient de corrélation $=+0,75$ ), soit le groupe d'âge le plus directement impliqué dans la migration de périurbanisation ${ }^{12}$. C'est ce qui explique le rajeunissement modéré lié aux migrations internes dans les communes rurales luxembourgeoises et ardennaises : l'attractivité de jeunes adultes et de leur(s) enfant(s) contrebalance l'impact positif sur le vieillissement qu'entraînent automatiquement les bilans migratoires favorables des retraités.

En d'autres termes, les migrations internes, par leur sélectivité selon l'âge et par extension selon le type de ménage, renforcent les contrastes démographiques entre les espaces ruraux considérés.

\section{Conclusion}

En regard des caractéristiques socio-économiques de la population, des variables environnementales ou encore de données sur la qualité de vie offerte par les communes, on peut affirmer que l'espace rural wallon est marqué par la diversité. Celle-ci se manifeste notamment par le rythme 
des évolutions démographiques. En moyenne, tous les types de communes rurales enregistrent, au cours de la dernière décennie, au moins, une croissance de leur population. L'intensité de celle-ci est bien évidemment variable, de très forte dans les milieux périurbains à très modérée dans les communes semi-industrielles de l'ancien axe industriel wallon. Les différences de comportements et de caractéristiques démographiques sont aussi significatives. Ainsi, dans les zones socio-économiquement plus défavorisées, tel que le semi-industriel ou le rural hennuyer, l'espérance de vie des populations est plus basse et la part relative des ménages monoparentaux et d'isolés - des types de ménages généralement définis comme « à risque » (Boulanger et al., 1997) - est en moyenne plus élevée. À l'opposé du gradient social, figurent les communes périurbaines, milieu privilégié à bien des égards.

Le rôle des migrations internes dans la structuration sociodémographique des espaces ruraux n'est pas négligeable. L'attractivité migratoire dépend des atouts offerts par une commune. Or, l'appréciation de ceux-ci est fonction de l'âge, du type de ménage, ou plus globalement, du cycle de vie des individus et des familles. Ainsi, la stratégie résidentielle dans le choix de la localisation pourra varier selon que l'on fasse partie d'un jeune couple avec enfants ou d'un ménage de retraités. Pour tous les groupes ruraux considérés, le bilan migratoire enregistré au cours de ces dernières années est positif, mais il varie sensiblement en fonction de l'âge. Les atouts environnementaux des espaces ruraux ardennais et luxembourgeois constituent de puissants motifs attractifs pour les personnes plus âgées. De même, la localisation à distance raisonnable des agglomérations urbaines, des bassins d'emploi et des voies de communication rapides qui caractérise les espaces périurbains est un facteur déterminant dans le choix résidentiel des couples actifs de 30-45 ans avec enfants.

Si la qualité de vie, l'environnement, la localisation ou encore les services offerts par une commune jouent un rôle important sur son attractivité migratoire, on peut également considérer que la migration et ses dimensions sélectives peuvent influencer, positivement ou négativement, la situation de la commune. Principal moteur de la croissance démographique locale, la fréquence et la répartition selon l'âge et le type de ménage des migrations peuvent modifier, peu ou prou, les caractéristiques démographiques, socio-économiques, culturelles, voire environnementales, des milieux d'accueil. Sous la pression notamment des marchés foncier et immobilier, les migrations contribuent aussi à une ségrégation sociale de plus en plus marquée des espaces. Ainsi, le « droit d'entrée » dans les espaces périurbains est aujourd'hui très sélectif. Les opportunités de s’y installer ou de s'y maintenir se réduisent de plus en plus, entraînant un tri social des espaces et un confinement de certains groupes sociaux dans des zones socioéconomiques dépréciées et retirées.

Annexe 1 : Valeurs propres des 5 premiers facteurs

Egeinvalues for the 5 th first factors

\begin{tabular}{|c|c|c|c|}
\hline & \multicolumn{3}{|c|}{ Valeurs propres initiales } \\
\hline Axes & Total & \% de la variance & \% cumulés \\
\hline 1 & 5,42 & 30,13 & 30,13 \\
2 & 4,17 & 23,18 & 53,31 \\
3 & 2,16 & 12,00 & 65,31 \\
4 & 1,76 & 9,77 & 75,08 \\
5 & 1,12 & 6,24 & 81,32 \\
\hline
\end{tabular}


Annexe 2 : Coefficient de corrélation entre les différentes variables selon les 5 axes retenus Correlation coefficient between covariates and the 5 factors

\begin{tabular}{|l|c|c|c|c|c|}
\cline { 2 - 5 } \multicolumn{1}{l|}{} & Axe 1 & Axe 2 & Axe 3 & Axe 4 & Axe 5 \\
\hline Émissions de particules en suspension & 0,11 & 0,19 & 0,87 & $-0,11$ & $-0,25$ \\
\hline Émissions acides & $-0,08$ & $-0,02$ & 0,85 & 0,03 & $-0,14$ \\
\hline Proportion de terres agricoles & 0,04 & 0,00 & $-0,19$ & 0,94 & $-0,05$ \\
\hline Proportion de bois & $-0,11$ & $-0,17$ & $-0,28$ & $-0,88$ & 0,15 \\
\hline $\begin{array}{l}\text { Indice de satisfaction quant aux espaces } \\
\text { verts }\end{array}$ & 0,26 & 0,06 & $-0,21$ & $-0,42$ & 0,77 \\
\hline Indice de satisfaction global & 0,32 & $-0,13$ & $-0,44$ & $-0,40$ & 0,58 \\
\hline Revenu médian & 0,73 & 0,14 & 0,12 & 0,31 & 0,07 \\
\hline Coût des habitations & 0,85 & 0,20 & 0,05 & 0,06 & 0,20 \\
\hline Coût des terrains à bâtir & 0,66 & 0,31 & 0,30 & 0,17 & $-0,01$ \\
\hline Taux de chômage & $-0,81$ & 0,15 & 0,26 & 0,19 & $-0,01$ \\
\hline Absence de chauffage central & $-0,82$ & $-0,05$ & 0,05 & 0,28 & 0,22 \\
\hline Connexion Internet & 0,89 & 0,09 & 0,17 & 0,11 & 0,23 \\
\hline $\begin{array}{l}\text { Indice de satisfaction quant à l'offre de } \\
\text { services de santé }\end{array}$ & $-0,04$ & 0,86 & 0,19 & 0,18 & $-0,10$ \\
\hline $\begin{array}{l}\text { Indice de satisfaction quant à l'offre de } \\
\text { transport en commun }\end{array}$ & 0,05 & 0,42 & 0,21 & $-0,08$ & $-0,72$ \\
\hline $\begin{array}{l}\text { Indice de satisfaction quant à l'offre de } \\
\text { commerces }\end{array}$ & 0,08 & 0,76 & 0,20 & $-0,16$ & $-0,48$ \\
\hline $\begin{array}{l}\text { Indice de satisfaction quant à l'offre de } \\
\text { services administratifs }\end{array}$ & 0,23 & 0,77 & 0,01 & 0,16 & 0,22 \\
\hline $\begin{array}{l}\text { Indice de satisfaction quant à la présence } \\
\text { de professions libérales }\end{array}$ & 0,17 & 0,89 & 0,10 & $-0,03$ & $-0,22$ \\
\hline Densité de l'habitat & 0,26 & 0,32 & 0,79 & 0,17 & $-0,06$ \\
\hline
\end{tabular}


Annexe 3 : Caractéristiques des 6 groupes retenus (moyennes)

\section{The 6 cluster characteristics (means)}

\begin{tabular}{|c|c|c|c|c|c|c|c|}
\hline Variables & $\begin{array}{c}\text { Semi- } \\
\text { industriel }\end{array}$ & Périurbain & $\begin{array}{l}\text { Rural } \\
\text { Lux. }\end{array}$ & $\begin{array}{c}\text { Rural } \\
\text { ardennais }\end{array}$ & $\begin{array}{c}\text { Rural } \\
\text { hennuyer }\end{array}$ & $\begin{array}{c}\text { Périurbain } \\
\text { nouveau }\end{array}$ & Ensemble \\
\hline $\begin{array}{l}\text { Émissions de particules en } \\
\text { suspension (1996) (tonnes } / \mathrm{km}^{2} \text { ) }\end{array}$ & 10,795 & 7,000 & 3,861 & 3,724 & 4,839 & 3,088 & 5,487 \\
\hline Émissions acides (1996) (tAéq/km²) & 0,848 & 0,332 & 0,196 & 0,165 & 0,331 & 0,262 & 0,341 \\
\hline $\begin{array}{l}\text { Superficie des terrains bâtis (2002) } \\
\left({ }^{*} 10 \mathrm{~m}^{2}\right)\end{array}$ & 125 & 109 & 25 & 26 & 61 & 55 & 62 \\
\hline $\begin{array}{l}\text { Superficie des terres agricoles (2002) } \\
\left(* 10 \mathrm{~m}^{2}\right)\end{array}$ & 480 & 583 & 449 & 376 & 696 & 730 & 514 \\
\hline $\begin{array}{l}\text { Superficie des terrains boisés (2002) } \\
\left({ }^{*} 10 \mathrm{~m}^{2}\right)\end{array}$ & 127 & 150 & 414 & 479 & 104 & 105 & 274 \\
\hline $\begin{array}{l}\text { Revenu médian par déclaration (euro) } \\
\text { (2002) }\end{array}$ & 17614 & 20718 & 17505 & 17186 & 17893 & 19179 & 18127 \\
\hline $\begin{array}{l}\text { Coût moyen des habitations (2002) } \\
\left({ }^{*} 1000 \text { euros }\right)\end{array}$ & 67,9 & 105,2 & 74,3 & 74,5 & 68,4 & 82,8 & 78,0 \\
\hline $\begin{array}{l}\text { Coût moyen des terrains à bâtir } \\
(2002)\left(\text { euro } / \mathrm{m}^{2}\right)\end{array}$ & 27,7 & 56,1 & 19,3 & 18,7 & 27,7 & 28,4 & 27,9 \\
\hline Taux de chômage (\%) (2001) & 22,0 & 10,5 & 11,2 & 17,6 & 21,0 & 14,8 & 16,5 \\
\hline $\begin{array}{l}\text { Proportion de logement sans } \\
\text { chauffage central (\%) (2001) }\end{array}$ & 32,4 & 20,1 & 21,0 & 32,3 & 34,3 & 28,9 & 28,8 \\
\hline $\begin{array}{l}\text { Proportion de logements disposant } \\
\text { d'une connexion Internet }(\%)(2001)\end{array}$ & 23,0 & 37,1 & 24,5 & 22,2 & 21,6 & 26,9 & 25,3 \\
\hline $\begin{array}{l}\text { Indice de satisfaction quant à l'offre } \\
\text { de services de santé } \\
(2001)\end{array}$ & 117 & 118 & 103 & 109 & 116 & 117 & 113 \\
\hline $\begin{array}{l}\text { Indice de satisfaction quant à l'offre } \\
\text { de transport en commun (2001) }\end{array}$ & 80 & 61 & 71 & 60 & 73 & 65 & 67 \\
\hline $\begin{array}{l}\text { Indice de satisfaction quant aux } \\
\text { espaces verts (2001) }\end{array}$ & 94 & 121 & 107 & 125 & 96 & 113 & 112 \\
\hline $\begin{array}{l}\text { Indice de satisfaction quant à l'offre } \\
\text { de commerces (2001) }\end{array}$ & 80 & 74 & 70 & 67 & 73 & 68 & 72 \\
\hline $\begin{array}{l}\text { Indice de satisfaction quant à l'offre } \\
\text { de services administratifs (2001) }\end{array}$ & 96 & 103 & 87 & 95 & 94 & 105 & 96 \\
\hline $\begin{array}{l}\text { Indice de satisfaction quant à la } \\
\text { présence de professions libérales } \\
(2001)\end{array}$ & 85 & 91 & 80 & 81 & 84 & 86 & 84 \\
\hline Indice de satisfaction global (2001) & 114 & 137 & 138 & 143 & 122 & 135 & 133 \\
\hline
\end{tabular}

Les indices de satisfaction ont été calculé par l'Institut national de Statistique : plus la valeur de cet indice est supérieure à 100 , plus la perception des personnes interrogées est positive, et inversement lorsque l'indice est inférieur à 100. 
Annexe 4 : Présentation de la méthode de calcul de l'impact des migrations internes sur l'évolution de l'âge moyen d'une population

Method for estimating internal migration impact on the mean age evolution in a local population.

Chaque personne appartenant, en 2002, à la population étudiée, peut être caractérisée selon son lieu de résidence (la commune) 10 ans auparavant. Pour les enfants de moins de 10 ans en 2002, soit ceux nés pendant la période d'observation, on comparera le lieu de résidence de la mère à leur naissance avec le lieu où ils résident en 2002.

Cela permettra de distinguer au niveau de chaque commune :

- les non migrants, soit les personnes qui résident dans la même commune en 1992 et en 2002 (pour les enfants de moins de 10 ans, il s'agira des individus dont la commune de résidence de la mère à leur naissance est celle où ils résident en 2002);

- les entrants, soit les personnes présentes dans la commune en 2002 et absentes de cette commune en 1992. On distinguera les entrants internes ou externes selon que leur lieu de résidence en 1992 est une autre commune belge ou situé à l'étranger (pour les enfants de moins de dix ans, les entrants seront les individus présents en 2002 mais dont la commune de résidence de la mère à leur naissance est différente);

- les sortants, soit les personnes présentes dans la commune en 1992 et absentes de cette commune en 2002. On distinguera les sortants internes ou externes selon qu'ils résident toujours en Belgique ou à l'étranger (pour les enfants de moins de dix ans, les sortants seront les individus dont la mère résidait dans la commune à leur naissance et qui résident ailleurs en 2002).

On calculera les indices suivants :

- l'effectif de la population en 1992 soit $\mathrm{P}(92)$;

- l'âge moyen de la population de la commune en 1992 soit x(92);

- l'effectif de la population en 2002 soit $\mathrm{P}(02)$;

- l'âge moyen de la population de la commune en 2002 soit $\mathrm{x}(02)$;

- l'effectif des entrants internes dans la commune soit E;

- l'âge moyen des entrants internes soit $\mathrm{x}(\mathrm{E})$;

- l'effectif des sortants internes dans la commune soit S;

- l'âge moyen des sortants internes soit $\mathrm{x}(\mathrm{S})$.

L'évolution d'une population dépend de plusieurs facteurs que l'on peut résumer comme suit :

$\mathbf{P}(02)=\mathrm{P}(92)+(\mathrm{E}-\mathrm{S})+(\mathrm{N}-\mathrm{D})+(\mathrm{Ei}-\mathrm{Si})$

ou

$\mathrm{N}$ est l'effectif des naissances;

$\mathrm{D}$ est l'effectif des décès;

Ei est l'effectif des entrants par migration internationale;

Si est l'effectif des sortants par migration internationale.

Sans mouvement naturel ni migrations internationales, on peut écrire :

$P(02)=P(92)+(E-S)$

Dans cette hypothèse, l'âge moyen de la population de cette commune en 2002 pourrait s'exprimer comme suit :

$$
\mathbf{x}(02)=\frac{\{\mathbf{P}(92) *(\mathbf{x}(92)+10)+\mathbf{E} * \mathbf{x}(\mathbf{E})-\mathbf{S} * \mathbf{x}(\mathbf{S})\}}{\mathbf{P}(\mathbf{9 2})+\mathbf{E}-\mathbf{S}}
$$


Cette équation (1) peut s'écrire :

$\mathbf{x}(\mathbf{0 2})=\frac{\{\mathbf{P}(\mathbf{9 2})+\mathbf{E}-\mathbf{S}\} *\{\mathbf{x}(\mathbf{9 2})+\mathbf{1 0}\}+\mathrm{E} *\{\mathbf{x}(\mathbf{E})-\mathbf{x}(\mathbf{9 2})-10\}-\mathrm{S}^{*}\{\mathbf{x}(\mathbf{S})-\mathbf{x}(\mathbf{9 2})-10\}}{\mathrm{P}(92)+\mathrm{E}-\mathrm{S}}$

ou

$$
\{\mathbf{x}(02)-\mathbf{x}(\mathbf{9 2})\}-10=\frac{E^{*}\{\mathbf{x}(\mathbf{E})-\mathbf{x}(\mathbf{9 2})-10\}-S^{*}\{\mathbf{x}(\mathbf{S})-\mathbf{x}(\mathbf{9 2})-10\}}{P(92)+E-S}
$$

Ceci exprime l'impact des mouvements migratoires internes sur l'évolution de l'âge moyen de la population. La différence entre $\mathrm{x}(02)$ et $\mathrm{x}(92)$ doit être diminuée de 10 ans, car en l'absence de tout mouvement migratoire et sous les conditions d'absence de mouvement naturel et de migration internationale, l'âge moyen augmenterait de 10 années.

Tout comme pour les entrants et les sortants, on peut estimer l'impact du mouvement naturel sur l'évolution de l'âge moyen en l'absence de migrations.

De la sorte, on décompose l'âge moyen d'une population en quatre termes : le premier représente l'effet du mouvement naturel, le deuxième globalise l'impact des mouvements migratoires internes, le troisième est l'effet des migrations internationales et le quatrième terme résiduel qui mesure l'impact de l'interaction entre mouvements naturel et migratoire. De fait, on ne peut ignorer l'effet induit des mouvements migratoires sur le nombre de naissances puisque une part de celles-ci sont dues ou perdues par la migration de leurs parents.

On peut donc écrire la formule comme suit :

$\{\mathrm{x}(02)-\mathrm{x}(92)\}=10+\{\mathrm{x}(02)-\mathrm{x}(92)\}_{\text {naturel }}+\{\mathrm{x}(02)-\mathrm{x}(92)\}_{\text {mig interne }}+\{\mathrm{x}(02)-\mathrm{x}(92)\}_{\text {mig internationale }}+$ résidu

On peut donc isoler pour chaque commune, chacun des termes. Ceux-ci étant calculés par rapport aux mêmes populations de référence, les résultats sont strictement comparables et parfaitement additifs. Dès lors, en sommant les trois impacts et le résidu (celui-ci exprime l'interaction entre le mouvement naturel et les migrations) et en y ajoutant les 10 années du vieillissement synchronisé, on obtient l'évolution de l'âge moyen tel que calculé par différence entre 2002 et 1992.

\section{Bibliographie}

Arbonville (D.), 2006. - «Évaluer les implications des évolutions sociodémographiques sur l'habitat en Europe », dans Bonvalet (C.), Arbonville (D.) (dir.), Quelles familles? Quels logements? La France et l'Europe du Sud, Les Cahiers de INED, n 157, p. 41-66.

Direction générale des Ressources naturelles et de l'Environnement, 1998. - Atlas de l'air de la Wallonie, Ministère de la Région Wallonne, Namur, 36 p.

BACCAÏNI (B.), 2000. - « L'espace rural rhônalpin. Dynamisme démographique et migrations résidentielles », Espace, Populations, Sociétés, n 3, p. 69-88.

Boulanger (P.-M.), Lambert (A.), Deboosere (P.), Lesthaeghe (R.) et Surkyn (J.), 1997. - Ménages et familles, monographie 4 du recensement de la population et des logements de 1991, INS-SSTC, Bruxelles, $236 \mathrm{p}$.

Champion (T.), 2000. - « The continuing urban-rural population movement in Britain: trends, patterns, significance ", Espace, Populations, Sociétés, n³ 3, p. 37-51.

DATAR, 2003. - Quelle France rurale pour 2020? Contribution à une nowvelle politique de développement rural durable, Étude prospective de la Datar, Paris, 59 p.

DE BIE (T.), 1996. - « Habiter... à prix d'or », dans Passé, présent du Brabant Wallon, s.l., p. 118-135. 
EggerickX (T.) et CAPron (C.), 2001. - «Rurbanisation et périurbanisation dans le centre de la Wallonie. Une approche socio-démographique », Espace, Populations, Sociétés, n ${ }^{1}$, p. 123-137.

Eggerickx (T.), Capron (C.), Hermia (J.-P.), 2002a. - « Les nouvelles dynamiques de peuplement dans les zones frontalières en Wallonie », Espace, Populations, Sociétés, n 1-2, p. 83-98.

Eggerickx (T.), Capron (C.), Hermia (J.-P.), Oris (M.), 2002b. - Démographie et développement durable. Migrations et fractures socio-démographiques en Wallonie (1990-2000), SSTC, Liège/Louvain-la-Neuve, 208 p. +45 cartes.

Hermia (J.-P.), Eggerickx (T.) et SAnderson (J.-P.), 2005. - « Frontières et fragmentation démographique de l'espace périurbain bruxellois », dans Arlaud (S.), Jean (Y.), Royoux (D.) (dir.), Rural-urbain. Nouveaux liens, nouvelles frontières, Rennes, PUR, p. 123-136.

Houillon (V.) et Thomsin (L.), 2000. - « Définitions du rural et de l'urbain dans quelques pays européens », Espace, Populations, Sociétés, n 3, p. 195-200.

LuginbüHL (Y.) (dir.), 2007. - Nouvelles urbanités, nouvelles ruralités en Europe, Peter Lang, Bruxelles, $532 \mathrm{p}$.

Maurin (E.), 2004. - Le ghetto français. Enquête sur le séparatisme social, La république des idées, s.l., $96 \mathrm{p.}$

Oris (M.), 2000. - «Périurbanisation et rurbanisation en Wallonie », Les Cahiers de l'urbanisme, n² 28-29, p. $56-58$.

Perrier-Cornet (P.), 2003. - «Quelles perspectives pour les campagnes françaises? », Ceras-revue Projet, $\mathrm{n}^{\circ} 274,7 \mathrm{p}$.

RAPPORT POLIWA 1977. - État démographique de la Wallonie et éléments pour une politique de population, Centre d'étude de la population et de la famille, Bruxelles, $412 \mathrm{p}$.

Van der Haegen (H.), Van Hecke (E.), Juchtmans (G.), 1996. - « Les régions urbaines belges en 1991 », Études Statistiques, n 104 , p. 3-42.

Van Hecke (E.) et Savenberg (S.), 2002. - «Suburbanisation et développement durable », Espace, Populations, Sociétés, n 1-2, p. 25-36.

Cet article a été reçu le 30 novembre 2006 et définitivement accepté le 20 décembre 2007. 